\title{
Dependence of spatial filtering by spike timing dependent synaptic plasticity on learning window
}

Kazuhisa Fujita

From Twentieth Annual Computational Neuroscience Meeting: CNS*2011

Stockholm, Sweden. 23-28 July 2011

Spike timing dependent synaptic plasticity (STDP) plays an important role in temporal information processing. Whereas our result of the previous study showed that an interconnected network with STDP plays a role in spatial information processing [1]. The role is spatial filtering. In the present study, we studied the effect of learing window of STDP on spatial filtering by the interconnected network with STDP using computer simulation.

STDP learning is domated by learning window. Various types of learning window of STDP are found in various brain. In a hippocampus, a post-before-presynaptic spike pair couses synaptic efficacy to strengthen and a pre-before-postsynaptic spike pair courses synaptic efficacy to weaken (here we called this type learning "hippocampal type learning"). In a electrosensory lobe of an electric fish, the learning of STDP is reverse of hippocampal type learning (here we called "electric fish type learning").

Using the learning window of hippocampal type, the network acted as spatial high-contrast filtering. Left figure of Fig.3 shows input image. Neuron on white and grey area receives high and low magnitude of input current, respectively. Middle figure in Fig.3 shows output image from the network. The output image means firing count of neurons of the network from $9000 \mathrm{msec}$ to
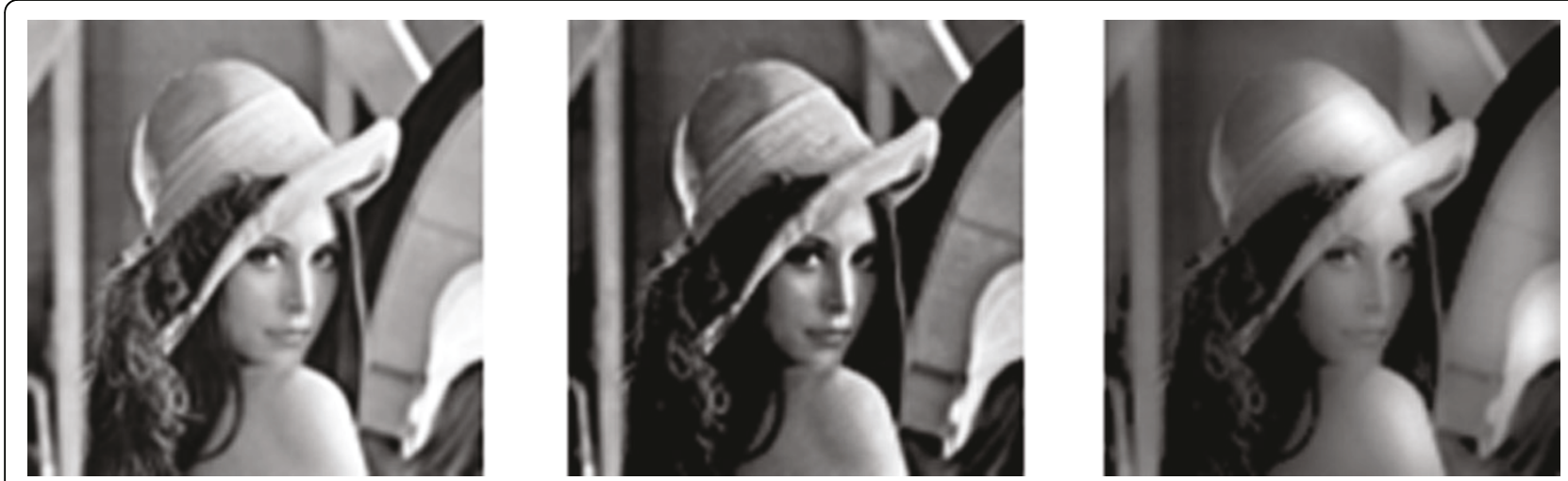

Figure 1 Left figure is an input image. Middle figure is output of the network with STDP applied hippocampal type learning window. Right figure is output of the network with STDP applied electric fish type learning window.

Correspondence: kazu@spikingneuron.net

Department of Computer and Information Engineering, Tsuyama National College of Technology, Japan 
$10000 \mathrm{msec}$. In this case, firing count of neurons which received lower and higher input is lower and larger, respectively. Thus the network with STDP functioned as spatial high contrast filtering using learning window of hippocampal type .

Using the learning window of electric fish type, the network acted as spatial low-pass filter. Left figure of Fig. 3 shows input image. Right figure of Fig.3 shows output image from the network. The output image means firing count of neurons of the network from $9000 \mathrm{msec}$ to $10000 \mathrm{msec}$. In this case, spatial high frequency component was reduced and spatial low frequency component is enhanced in output. Thus the network functioned as spatial low-pass filter using learning window of electric fish type.

A function of spatial filtering was provided by an interconnected network with STDP. The function changed according to a type of learning window of STDP. In the preset study, we proposed two type of spatial filtering using two type of learning window. The spatial filtering was achieved by dynamically changing connection of the network based on STDP. The results of this study suggested that the interconnected network with STDP might provide appropriate spatial filtering for an optional image from changing network connection according to the image.

Published: 18 July 2011

Reference

1. Fujita K: Spatial feature extraction by spike timing dependent synaptic modification. Lecture Notes in Computer Science 2010, 6443:148-154.

doi:10.1186/1471-2202-12-S1-P113

Cite this article as: Fujita: Dependence of spatial filtering by spike timing dependent synaptic plasticity on learning window. $B M C$ Neuroscience 2011 12(Suppl 1):P113.

\section{Submit your next manuscript to BioMed Central} and take full advantage of:

- Convenient online submission

- Thorough peer review

- No space constraints or color figure charges

- Immediate publication on acceptance

- Inclusion in PubMed, CAS, Scopus and Google Scholar

- Research which is freely available for redistribution

Submit your manuscript at www.biomedcentral.com/submit 